\title{
Secondary Heamorrhage After Cold Steel Tonsillectomy: An Audit Performed in Shalamar Hospital \\ ${ }^{1}$ Arshadullah Afridi, ${ }^{2}$ Sana Tariq, ${ }^{3}$ Muhammad Dawood, ${ }^{4}$ Sarwat Iqbal, ${ }^{5}$ Usna Athar, ${ }^{1}$ Aisha Hanif \\ ${ }^{1}$ Department of ENT, Shalamar Hospital, Lahore \\ ${ }^{2}$ Department of Bioethics, Shalamar Medical \& Dental College, Lahore \\ ${ }^{3}$ Department of Forensic Medicine, Shalamar Hospital, Lahore \\ ${ }^{4}$ Department of Medicine, Shalamar Hospital, Lahore \\ ${ }^{5}$ Department of Community Medicine, Shalamar Hospital, Lahore
}

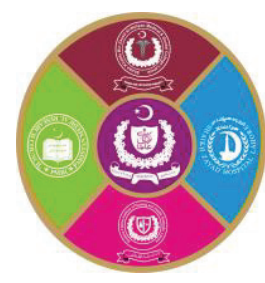

\begin{abstract}
Introduction: Tonsillectomy is a common surgical procedure in which post tonsillectomy heamorrhage remains the most serious complication. The cold steel dissection method is the oldest and safest method used to perform tonsillectomies. Aims \& Objectives: This study was done to see the frequency of posttonsillectomy heamorrhage with cold steel dissection method. It was a clinical audit performed retrospectively to compare our results with International standards. Place and duration of study: Our clinical audit was conducted at Otolaryngology department in Shalamar Hospital, Lahore, Pakistan. This study was conducted in a period of 6 months. Material \& Methods: The charts of patients $(n=344)$ that underwent tonsillectomy at Shalamar Hospital from January 2016 till December 2019 were retrospectively viewed, in a cross-sectional, pilot study, using convenience sampling technique. All the tonsillectomy procedures were performed using the cold steel dissection method. Frequencies were recorded and chi-square test was applied. Results: The complication of secondary heamorrhage occurred in 7 patients and the frequency was calculated as $2 \%$. A significant difference $(\mathrm{P}=0.01)$ of age was found in patients suffering from secondary heamorrhage. The incidence was lower in patients below 18 years of age $(0.7 \%)$ as compared to patients above 18 years of age (7.14\%). The rate of heamorrhage was found to be slightly higher in males than in females $(2.6 \%$ and $1.31 \%$ respectively). Conclusion: We conclude that the rate of secondary heamorrhage in our institution is up to the rate reported in literature. Different hot techniques for tonsillectomy should also be performed in clinical settings and compared with the standard cold steel dissection tonsillectomy.
\end{abstract}

Key words: Tonsillectomy, Adeno-tonsillectomy, Secondary Heamorrhage, Cold Steel Method

\section{INTRODUCTION}

$\mathrm{T}$ onsillectomy is one of the most common and frequently performed surgical procedures in otolaryngology practices in which both palatine tonsils are removed from the throat. In some cases, the adenoid may also be removed which is then termed as "adeno-tonsillectomy". ${ }^{1}$ It is often categorized as low risk operation with quite a few chances of complications whilst the post tonsillectomy heamorrhages remains the most serious complication. ${ }^{2}$ Post-tonsillectomy heamorrhages are fairly divided into two main categories: Primary heamorrhage in which postoperative bleeding occurs within 24 hours after the surgery while the patient is in hospital, and the
Secondary heamorrhage which occurs up to 2 weeks post operatively. ${ }^{3}$

To reduce both the intra and post-operative compilations, and to minimize the surgery duration and morbidity, the tonsillectomy techniques are evolving constantly. ${ }^{4}$ Tonsillectomy can be performed by various methods which are divided into two major categories including 'cold' and 'hot' methods. Dissection and snare, Guillotine Method, Intra-capsular, Harmonic Scalpel, Plasma mediated ablation technique and Cryosurgical techniques are the cold methods to perform tonsillectomy. ${ }^{5}$ The hot methods include; Electrocautery, Laser Tonsillectomy using $\left(\mathrm{CO}_{2}\right.$ or KTP), Colbation tonsillectomy and Radio frequency. ${ }^{6}$ However, it cannot be clearly stated that which of these techniques shows ascendancy over the other. ${ }^{4}$ 
Primarily, the main indications for tonsillectomy are based on obstructive and infectious causes. A surgery is required when there are periodic episodes of throat infections whose record is maintained through proper documentation. The physician might recommend tonsillectomy if the frequency of throat infection is of seven episodes per year, five episodes per 2 years or at least three episodes per 3 year, including the history ofPFAPA syndrome ( Periodic fever, Aphthous stomatitis, Pharyngitis and Cervical adenitis), peri-tonsillar abscesses multiple antibiotic allergy and drug intolerance. ${ }^{1}$ Other indicators such as Obstructive Sleep Apnoea syndrome, Dysphonia, Dysphagia, Suspected Malignancy, Chronic Tonsillitis, Halitosis, Tonsilloliths are also the reasons for considering tonsillectomy. ${ }^{1,7}$

The cold steel dissection method was introduced about 100 years ago, in which the blunt dissection is done involving a metal instrument and the subsequent bleeding is controlled with the help of gauze. ${ }^{8}$ The cold steel method is often considered as the gold standard because every other innovative techniques for tonsillectomy are compared with it. ${ }^{9}$ It is also suggested that the young ENT trainees must be taught to perform tonsillectomy with cold method first. ${ }^{10}$

The primary rationale behind this institutional review and analysis is to determine the frequency of secondary heamorrhages over the period of four years in Shalamar Medical and Dental College and Shalamar Hospital. This will help in ascertaining the risk factors associated with cold steel dissection tonsillectomy. This clinical audit may prove beneficial as a scaffold for comparing the results with different studies in order to monitor and modify our practices. This would help in improving patients' clinical outcomes. Clinical audits and reviews are essential in health care services. As clinical practices and guidelines are changing with time and the advent of technology, surgeons must put their work under a microscope and modify their techniques accordingly for a state-of-the-art health care provision.

\section{MATERIAL AND METHODS}

A retrospective, cross sectional study was conducted in Ear, Nose and Throat Department (ENT) of Shalamar Hospital. This study can be classified as a pilot audit conducted by the authors. Ethical approval was taken from the Institutional Review Board (IRB) at Shalamar Institute of Health Sciences (SIHS). After the IRB approval, the study was completed within 6 months. Since this is a clinical audit, so it needs no specific sample size calculation. Convenience sampling was used; the study was conducted in duration of 6 months.

The medical charts of the patients that underwent the tonsillectomy and/or Adeno- tonsillectomy were prepared dating from January 2016 till December 2019. The data of total of 344 cases was obtained from the Otolaryngology (ENT) department of SMDC which included both adults and children. The inclusion criteria was determined as patients with a history of recurrent attack of recurrent attacks of tonsillitis accompanied by clinical examination findings of high grade fever, palpable jugulodigastric lymph nodes, exudates on tonsil and absence of cough. All patients aged 3 years and above, who underwent the 'cold steel dissection' method for tonsillectomy were included. Patients having suspicion of malignancy or undergoing tonsillectomy as a part of uvulopalatoplasty were excluded. According to the set pattern of surgery, operations were performed on each patient and the surgical technique followed for tonsillectomy was 'cold steel dissection' specifically. The tonsils were removed using tonsillar dissector and haemostasis was secured by ligation and bipolar electro-cautery. Heamorrhage was monitored qualitatively in the post-operative phase during their stay in the hospital. The oropharyngeal cavity including the surgical site was examined every six hours using a tongue depressor and a light source to check for any possible bleeding. The patients were asked to report back to the hospital in case of any signs of bleeding, after discharge.

\section{Statistical analysis:}

The data of tonsillectomy cases was analyzed by using SPSS version 20. Frequency tables were formed. Chi-square test was applied for analytical comparison and $\mathrm{P}$-value was set as 0.05 with $95 \%$ Confidence Interval.

\section{RESULTS}

A total of 344 patients underwent tonsillectomy and adeno-tonsillectomy by the cold steel method only during January 2016 till December 2019. Demographic features and frequency of secondary heamorrhage were studied. Chi-square test was applied with the level of significance set at 0.05. Figure 1 shows the frequency of operations done according to the age of the patients. 


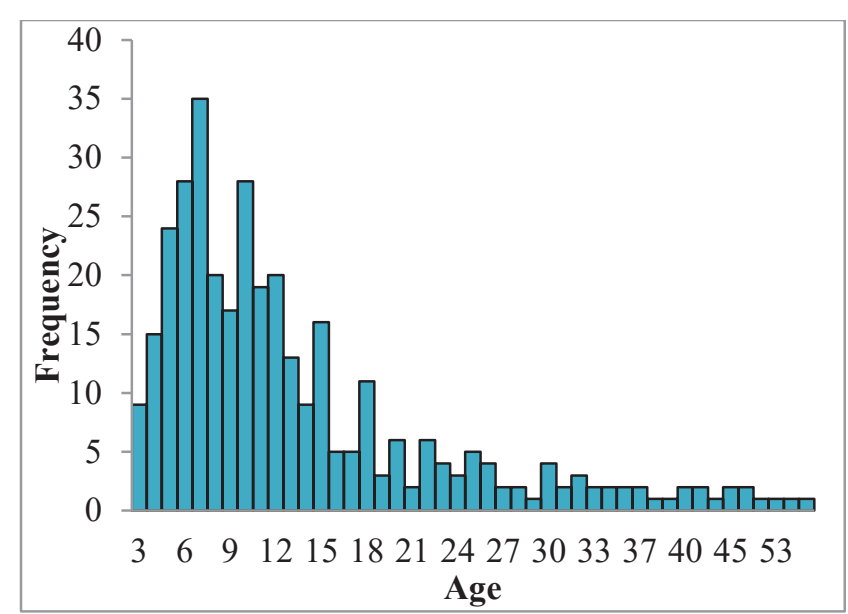

Fig-1: Frequency of tonsillectomy cases against the age of the patients.

Table-1 illustrates other demographic features as well as the results. Out of the total number of tonsillectomy cases, 337 cases did not experience secondary heamorrhage. Only 7 cases reported postoperative heamorrhage so the rate of secondary heamorrhage recorded was $2 \%$. There were zero cases reported in our hospital for mortality as a result of tonsillectomy.

\begin{tabular}{|c|c|}
\hline \multicolumn{2}{|l|}{ Age (in Years) } \\
\hline Mean & 13.66 \\
\hline Median & 10 \\
\hline Mode & 7 \\
\hline Std. Deviation & 10.11 \\
\hline Range & 52 \\
\hline Minimum & 3 \\
\hline Maximum & 55 \\
\hline \multicolumn{2}{|l|}{ Sex } \\
\hline Male & $192(55.8 \%)$ \\
\hline Female & $152(44.2 \%)$ \\
\hline \multicolumn{2}{|l|}{ Type of Surgery: } \\
\hline Adenotonsillectomy & $137(39.8 \%)$ \\
\hline Tonsillectomy & $207(60.2 \%)$ \\
\hline \multicolumn{2}{|c|}{ Rate of secondary haemorrhage } \\
\hline Haemorrhage & $7(2 \%)$ \\
\hline
\end{tabular}

Table-1: Characteristics of study participants undergoing tonsillectomy $(\mathrm{n}=344)$

Shalamar Institute of Health Sciences is a trust hospital where people from different financial backgrounds are treated. Fig-2 represents the statistics of 4 consecutive years starting from 2016, where the mode of payment for the number of different patients is categorized into 3 categories accordingly.

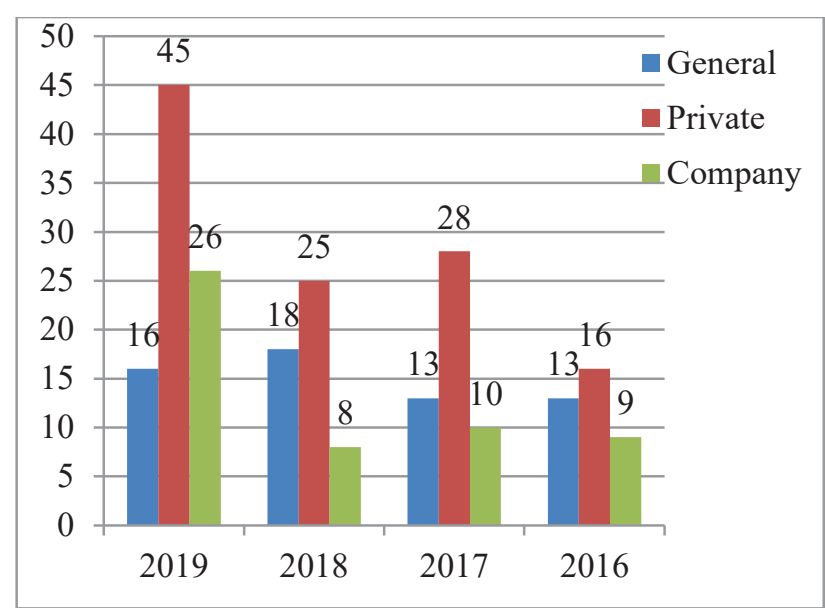

Fig-2: Comparison of 'General, Private \& Company' patients of tonsillectomy over past 4 Years.

The data of the patients were divided into two groups on the basis of their age. Our study shows that only $0.72 \%$ of patients under the years of 18 experienced secondary heamorrhage whereas for the patients older than 18 years of age the percentage was $7.14 \%$. The rate of secondary heamorrhage was higher for the patient with age more than 18 years and this result was statistically significant ( $P$ value $=0.01$ )

In our study, the rate of secondary heamorrhage in males is $2.6 \%$ and for females it is $1.31 \%$. There is no significant difference between male and female regarding the rate of secondary heamorrhage $(\mathrm{P}$ value $=0.401$ )

In our study, no statistically significant difference was recorded between adeno-tonsillectomy and tonsillectomy regarding the rate of secondary heamorrhage $(\mathrm{P}$ value $=0.869)$.

\section{DISCUSSION}

The rate of secondary heamorrhage in our study is $2 \%$ which is higher than the rates reported by O'Leary and Vorrath (1.85\%) but lower than described by Brick et al. 4.23\%. ${ }^{4,11}$ Harju and Numminen reported higher secondary heamorrhage rate $(12 \%)$ as 208 patients out of total 1734 patients who underwent tonsillectomy experienced secondary haemorrhage. ${ }^{12}$

When looking at the two groups divided on the basis of age, Our study showed similar results to the one done by Walker $\mathrm{P}$, in which post-tonsillectomy heamorrhage for 18 years old patients was $10.1 \%$ and age was defined as a highly statistically significant ( $\mathrm{P}$ value $=0.001$ ) risk factor for posttonsillectomy haemorrhage. ${ }^{13}$ The cold steel dissection method has been preferred in older children, adolescents and adults. ${ }^{9}$ 
In contrast to our findings, Ali et al. shows a high rate of post tonsillectomy bleeding $(6.68 \%)$ in and it was indicted that the bleeding rate was higher in males as compared to the females (7.66 and 5.9\% respectively). ${ }^{14}$

In another comparative study, the group of patients $(\mathrm{n}=40)$ subjected to the cold steel dissection tonsillectomy showed $2.5 \%$ of secondary heamorrhage rate. It was also indicated that the patients who underwent tonsillectomy with the cold steel method showed quick healing with lesser amount of pain as compared to the other group patients subjected to bipolar diathermy. ${ }^{15}$

Preforming tonsillectomy through the cold steel method is quite a common practice even in the United States of America as it is characterized as cheaper and safer. ${ }^{16}$ As the tissue healing is better in cold steel method, it is reported as less painful technique in the literature. ${ }^{17}$ Cold steel dissection method may have certain advantages but surgeons should be trained in several other techniques of tonsillectomy and should be permitted to use different methods for individual patients as well. Extreme precautions are required for cold dissection in order to avoid the vascular damage.

Regardless of the operative technique, the frequency of post-operative bleeding also depends upon the skills and experiences individual surgeon. ${ }^{18}$ It has been seen in a study that the complication of post-tonsillectomy heamorrhageis reportedly higher when young resident perform the coblation technique. Hence, when younger surgeons are to perform this specific technique, supervision and surveillance are extremely vital. ${ }^{19}$ To further emphasize this, a research described that the rate of post-tonsillectomy heamorrhage was not dependent on the method used specially when the consultants performed the surgery. ${ }^{20}$ Post-operative administration of dexamethasone was also seen to raise the frequency of heamorrhage after tonsillectomy. ${ }^{21}$ Male gender and age were also studied as some of the risk factors for increased post-tonsillectomy heamorrhage rates and returns to the operating table. ${ }^{22}$

The complication of post-tonsillectomy heamorrhage has high mortality and thus must be evaluated with priority. Some patients with this particular complication may be managed conservatively, but most of them need an intervention ranging from cautery to external carotid artery ligation. ${ }^{23}$ Rising rates of secondary heamorrhage after tonsillectomy can be reduced by employing quality improvement projects like the one used in Sweden which included hands-on workshops and supervision. ${ }^{24}$ Various quality assurance programs targeting the local hospitals and clinics can improve the clinical outcomes, which is an indicator of health care provision in a country. ${ }^{25}$ An important component of such quality improvement programs is clinical audit, much similar to the one performed by us. A regular audit and maintenance of a registry can provide clinicians and surgeons with evidence to modify practice guidelines.

\section{CONCLUSION}

There are many studies published showing the frequency of secondary heamorrhage after tonsillectomies but no study has been published containing data from Shalamar Institute of Health Sciences. It was seen in our pilot study that the frequency of secondary heamorrhagic complication was similar to what was reported in other peerreviewed publication. This in turn exhibits that standard techniques are being applied by our surgeons performing tonsillectomies. The introduction of Electronic Surgical Performa along with the manual notes has resulted in improving the quality of data maintenance for routinely performed tonsillectomies. As evident, there is a low amount of data available right now, over the years the reauditing for different factors will become systematic and easy.

\section{Limitations:}

There is a lot of literature available on this topic with the with sample size ranging in thousands. Since before 4 years the electronic data system did not exist, our study only comprises of records of the past 4 years hence the sample size is low. In other studies, the comparison of different tonsillectomy technique is analyzed. However, in Shalamar medical and dental college tonsillectomy is only performed with cold steel dissection method. The proper documentation of the initial indicators for performing tonsillectomy is also lacking.

\section{REFERENCES}

1. Mitchell RB, Archer SM, Ishman SL, Rosenfeld RM, Coles S, Finestone SA, et al. Clinical Practice Guideline: Tonsillectomy in Children (Update). 2019;(4)S1-S42.

2. Patel A, Foden N, Rachmanidou A. Is weekend surgery a risk factor for post-tonsillectomy haemorrhage? J Laryngol Otol. 2016; 130(8): 763-7.

3. Lechner M, Chandrasekharan D, Vithlani R, Sutton L, Grandidge C, Elmiyeh B. Evaluation 
of a newly introduced tonsillectomy operation record for the analysis of regional posttonsillectomy bleed data: a quality improvement project at the London North West Healthcare NHS Trust. BMJ Open Qual.2017;6(2):e000055

4. Brkic F, Mujic M, Umihanic S, Hrncic N, Goga A, Goretic E. Haemorrhage Rates After Two Commonly Used Tonsillectomy Methods: a Multicenter Study. Med Arch (Sarajevo, Bosnia Herzegovina). 2017; 71(2):119-21.

5. Walker RA, Syed ZA. Harmonic scalpel tonsillectomy versus electrocautery tonsillectomy: A comparative pilot study. Otolaryngol - Head Neck Surg. 2001; 125(5): 449-55.

6. Verma R, Verma RR, Verma RR. Tonsillectomy-Comparative Study of Various Techniques and Changing Trend. Indian $\mathrm{J}$ Otolaryngol Head Neck Surg.2017;69(4):549-58.

7. Moroco AE, Saadi RA, Wilson MN. Posttonsillectomy respiratory complications in children with sleep disordered breathing. Int $\mathrm{J}$ Pediatr Otorhinolaryngol. 2020; 131(October 2019):109852.

8. BAO-HNS, RCS. National Prospective Tonsillectomy Audit. Royal College of Surgeons of England. 2005. 1-50 p.

9. Blanchford H, Lowe D. Cold versus hot tonsillectomy: State of the art and recommendations. Orl. 2013; 75(3):136-41.

10. Söderman ACH, Odhagen E, Ericsson E, Hemlin C, Hultcrantz E, Sunnergren O, et al. Post-tonsillectomy haemorrhage rates are related to technique for dissection and for haemostasis. An analysis of 15734 patients in the National Tonsil Surgery Register in Sweden. Clin Otolaryngol. 2015; 40(3):248-54.

11. O'Leary S, Vorrath J. Postoperative bleeding after diathermy and dissection tonsillectomy. Laryngoscope. 2005; 115(4):591-4.

12. Harju T, Numminen J. Risk factors for secondary post-tonsillectomy haemorrhage following tonsillectomy with bipolar scissors: Four-year retrospective cohort study. J Laryngol Otol. 2017; 131(2):155-61.

13. Walker P, Gillies D. Post-tonsillectomy hemorrhage rates: Are they techniquedependent? Otolaryngol - Head Neck Surg. 2007; 136(4 SUPPL.).

14. Ali RB, Smyth D, Kane R, Donnelly M. Posttonsillectomy bleeding: A regional hospital experience. Ir J Med Sci. 2008; 177(4):297-301.

15. Chughtai A, Haq AU, Ullah S. Is the cold steel dissection method still the most effective method of tonsillectomy? 2016; 66(5).
16. Leinbach RF, Markwell SJ, Colliver JA, Lin SY. Hot versus cold tonsillectomy: A systematic review of the literature. Otolaryngol - Head Neck Surg. 2003; 129(4):360-4.

17. Aydin S, Taskin U, Altas B, Erdil M, Senturk T, Celebi S, et al. Post-tonsillectomy morbidities: Randomised, prospective controlled clinical trial of cold dissection versus thermal welding tonsillectomy. J Laryngol Otol.2014;128(2):163-5

18. Motta S, Testa D, Ferrillo B, Massimilla EA, Varriale R, Motta G, et al. Surgical techniques and post-tonsillectomy haemorrhage. 2017; 21(4):559-66.

19. Heidemann CH, Wallén M, Aakesson M, Skov $\mathrm{P}$, Kjeldsen AD, Godballe C. Post-tonsillectomy hemorrhage: assessment of risk factors with special attention to introduction of coblation technique. European Archives of Oto-rhinolaryngology. $2009 \mathrm{Jul}$ 1; 266(7):1011.

20. Hinton-Bayre AD, Noonan $K$, Ling $S$, Vijayasekaran S. Experience is more important than technology in paediatric post-tonsillectomy bleeding. The Journal of Laryngology \& Otology. 2017 Jul; 131(S2):S35-40.

21. Ordemann AG, Hartzog AJ, Seals SR, Spankovich C, Stringer SP. Is weight a predictive risk factor of postoperative tonsillectomy bleed? Laryngoscope investigative otolaryngology.2018Jun;3(3):238-43

22. Hinton-Bayre AD, Noonan $K$, Ling S, Vijayasekaran S. Experience is more important than technology in paediatric post-tonsillectomy bleeding. The Journal of Laryngology \& Otology. Cambridge University Press; 2017; 131(S2):S35-S40.

23. Abdel Rahman AA, Al-Morsy MA, Hussein MS. Incidence, Prevention and Management of Post Tonsillectomy Hemorrhage. The Egyptian Journal of Hospital Medicine. 2019 Jan 1; 74(5):1123-7.

24. Odhagen E, Sunnergren O, Söderman AC, Thor J, Stalfors J. Reducing post-tonsillectomy haemorrhage rates through a quality improvement project using a Swedish National quality register: a case study. European Archives of Oto-Rhino-Laryngology. 2018 Jun $1 ; 275(6): 1631-9$.

25. Kaplan HC, Provost LP, Froehle CM, Margolis PA. The Model for Understanding Success in Quality (MUSIQ): building a theory of context in healthcare quality improvement. BMJ Qual Saf. 2012; 21(1):13-20. 
The Authors:

Dr. Arshadullah Afridi

Assistant Professor,

Department of ENT,

Shalamar Hospital, Lahore.

Dr. Sana Tariq

Research Associate,

Department of Bioethics,

Shalamar Medical \& Dental College, Lahore.

Dr. Muhammad Dawood

Demonstrator,

Department of Forensic Medicine,

Shalamar Hospital, Lahore.

Dr. Sarwat Iqbal

Assistant Professor,

Department of Medicine,

Shalamar Hospital, Lahore.
Dr. Usna Athar

Demonstrator,

Department of Community Medicine,

Shalamar Hospital, Lahore.

Dr. Aisha Hanif

Medical Officer,

Department of ENT,

Shalamar Hospital, Lahore.

\section{Corresponding Author:}

Dr. Arshad Ullah Afridi

Assistant Professor,

Department of ENT,

Shalamar Hospital, Lahore.

E-mail: arshadullah@hotmail.com 